\title{
Huge pyogenic cervical cyst with endometriosis, developing 13 years after myomectomy at the lower uterine segment: a case report
}

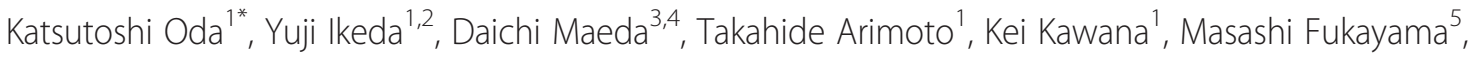
Yutaka Osuga ${ }^{1}$ and Tomoyuki Fujii ${ }^{1}$

\begin{abstract}
Background: Surgical site infections are potential complications following open myomectomy. These infections usually develop immediately after the surgery, and are most often located in the myometrium. Pyogenic cervical cysts are rare and have not been previously reported to occur at the site of myomectomy.

Case presentation: A 41-year-old nulligravida Japanese woman was referred to our hospital with a large cervical cyst (>15 cm in diameter). She had undergone a myomectomy 13 years previously, and the surgical site had extended to the endocervical gland. Standard blood tests did not show any evidence of inflammation. The patient underwent a total abdominal hysterectomy, which revealed that the cyst contained multiple components, including Escherichia coli, old blood, and evidence of endometriosis. A pathological review did not show malignant cells within the cyst. The pyogenic cyst originated from the upper anterior cervix, which was one of the sites involved in the previous myomectomy.

Conclusion: We reported a huge pyogenic cervical cyst exhibiting signs of endometriosis, in the vicinity of the uterine scar from the open myomectomy. The previous surgery and endometriosis might have contributed to the formation of this rare pyogenic cyst.
\end{abstract}

Keywords: Cervical pyogenic cyst, Uterine fibroid, Myomectomy scar, Endometriosis

\section{Background}

Uterine fibroids are commonly observed, affecting $>30 \%$ of women of reproductive age [1]. Fertility-sparing surgery (myomectomy) is one of the major treatment options for younger patients [2], although potential risks are associated with this treatment. Myomectomy is associated with a risk of recurrent uterine fibroids, pelvic adhesions, as well as complications at the incision site. Furthermore, myomectomy increases the risk of uterine rupture during labor, and commonly requires planned cesarean sections [3]. Unfortunately, post-cesarean section abscess formation has been reported at the site of uterine incision [4]. Moreover, intra-uterine infection may develop before or after cesarean section, and the

\footnotetext{
*Correspondence: katsutoshi-tky@umin.ac.jp

'Department of Obstetrics and Gynecology, The University of Tokyo, 7-3-1 Hongo, Bunkyo-ku, Tokyo 113-8655, Japan

Full list of author information is available at the end of the article
}

risk of surgical site infection has been reported to increase after cesarean section [5]. However, no studies have reported the development of pyogenic cervical cysts, without signs of infection, after myomectomy. In the present report, we describe a rare case of a pyogenic cervical cyst, further complicated by endometriosis, arising 13 years after open myomectomy.

\section{Case presentation}

A 41-year-old nulligravida Japanese woman was referred to our hospital (Kawakita General Hospital, Tokyo, Japan) due to the presence of a large cervical mass. Thirteen years previously, she had undergone myomectomy for the treatment of multiple uterine fibroids in the lower uterine segment, including one weighing $1.4 \mathrm{~kg}$. Penetration beyond the endocervical gland had occurred during resection, and the anterior endocervical canal had been opened 

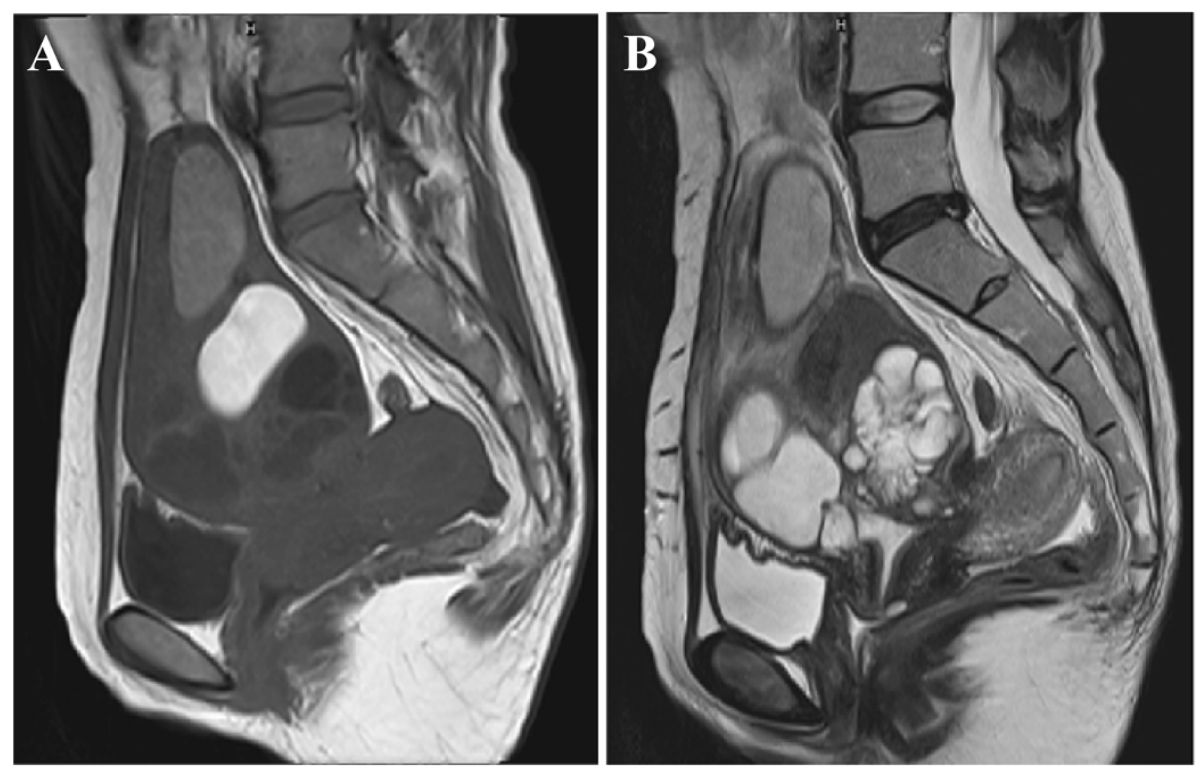

Figure 1 Magnetic resonance imaging (MRI) of the pyogenic cervical cyst. (A) a sagittal T1-weighted and (B) a sagittal T2-weighted imaging revealed a large cystic mass at the anterior cervix. The mass was a complex multilocular cyst, consisting of at least two components. The cranial part of the cyst contained two high-intensity cystic masses on the T1-weighted image, and the caudal part contained two multilocular masses that demonstrated low-intensity on the T1-weighted image and high-intensity on the T2-weighted image.

during the surgery. Her original follow-ups were approximately once a year at another hospital, where cancer screening was performed using transvaginal ultrasonography and cytology of the cervix and endometrium. Eight years after the original surgery, she had undergone pelvic magnetic resonance imaging (MRI), which did not detect any abnormal masses in the uterine body or cervix. Twelve years after the original surgery, she underwent transvaginal ultrasonography, and no abnormal masses were detected.

However, a pelvic examination, performed 13 years after her myomectomy, indicated marked enlargement of the cervix, which was suspected to be a cervical malignancy. The patient had experienced no symptoms prior to the examination, and given the lack of observed progression, we were unable to estimate the period over which the mass developed. Transvaginal ultrasonography indicated that the mass contained multilocular, hypoechoic lesions, with heterogeneous internal echogenicity. Furthermore, MRI confirmed the presence of a multilocular, irregularly shaped cystic mass (overall diameter, $>15 \mathrm{~cm}$ ) in the upper anterior cervix (Figure 1A and $\mathrm{B})$. Computed tomography (CT) did not show any evidence of enlarged lymph nodes, ascites, or a distant tumor. Blood tests did not show elevated levels of inflammation or tumor markers. The patient's white blood cell count was $6,000 / \mathrm{mL}$ and C-reactive protein level was $<0.3 \mathrm{mg} / \mathrm{dL}$. Levels of CA125, CA19-9, and squamous cell carcinoma antigen $(\mathrm{SCC})$ were $32.4 \mathrm{U} / \mathrm{mL}$,

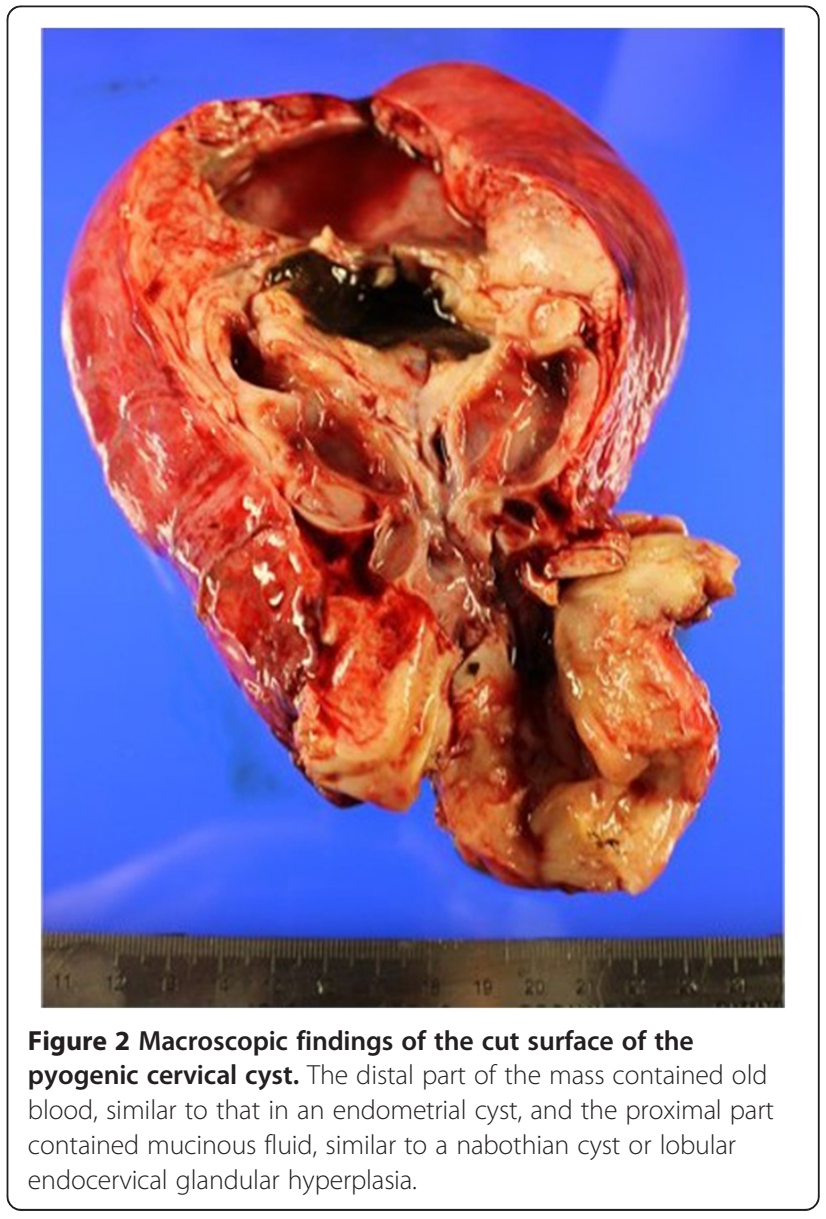


6.4 $\mathrm{U} / \mathrm{mL}$, and $1.4 \mathrm{U} / \mathrm{mL}$, respectively. Results from cytological examination of both the cervix and endometrium were negative for cancer.

Therefore, we suspected that the mass was either a degenerated uterine fibroid, lobular endocervical glandular hyperplasia, or cervical malignancy. We performed a total abdominal hysterectomy, and dense adhesions were observed in the pelvis, particularly around the cervical mass. Moreover, the cervix was markedly enlarged, given the mass in the anterior wall. However, no additional abscesses or ascites were detected in the abdomen. A macroscopic examination revealed that the cystic mass originated from the upper anterior cervix, which was one of the sites involved in the original myomectomy. The mass consisted of 2 components, one containing an abscess within the soft solid tissue, and another that was filled with old blood (similar to an endometrial cyst) (Figure 2).

Following culture of the abscess tissue, Escherichia coli was identified as the causative bacteria. Microscopic examination revealed the coexistence of endometriosis, and an abscess was confirmed within the cyst, although malignant cells were not identified. An additional pathological review was performed at the University of Tokyo, which confirmed the absence of atypical or malignant cells. Thus, we diagnosed the mass as a pyogenic cyst, with endometriosis, which most likely occurred at the site of the uterine scar.

\section{Discussion}

Surgical site infections are common complications of myomectomy, and the development of infection should be carefully examined, particularly in patients where the scar extends to the uterine cavity [6-8]. However, postoperative infections are typically early-onset, and the etiology of late-onset infections is not well understood.

One previous study has described how the vagina can act as a bacterial reservoir during the fecal-vaginal course of transmission in extraintestinal infections [9], which could hypothetically explain the late onset in the present case. However, we are uncertain when or how the infection developed, which precludes us from concluding that the pyogenic cyst was caused by late-onset infection secondary to the myomectomy. Another possibility is that the endometriosis was significantly associated with the infection, although it is surprising that no symptoms or inflammation markers were observed at the time of our initial diagnosis. This suggests that the infection did not occur immediately, and that the myomectomy scar was associated with the formation of endometriosis. Alternatively, a cervical endometriotic cyst might have progressed to pyogenic granuloma, or a gigantic cervical abscess might have developed de novo within the endometriosis. Patients should be carefully monitored for development of pyogenic cervical cysts; this case may indicate the currently unknown etiology of these cysts.

\section{Conclusions}

We have reported the rare case of a pyogenic cervical cyst, containing signs of endometriosis and $E$. coli infection, in the vicinity of the uterine scar from previous open myomectomy. The previous surgery might be associated with the endometriosis at the site of infection, and endometriosis and/or infection might have contributed to the formation of the cyst.

\section{Consent}

The patient gave written consent for the case report to be published.

\section{Abbreviations \\ CT: Computed tomography; MRI: Magnetic resonance imaging; SCC: Squamous cell carcinoma antigen.}

\section{Competing interests}

The authors declare that they have no competing interests.

\section{Authors' contributions}

$\mathrm{KO}$ and $\mathrm{YI}$ treated the case and wrote the manuscript. DM and MF diagnosed the case pathologically. TA, KK, YO, and TF contributed to the diagnosis, obtained informed consent, and determined the management of the case. All authors read and approved the final manuscript.

\section{Acknowledgments}

We thank Tsuneki Nagasaka, Iwao Hosoya, and Hirofumi Haraguchi for their support. This work was supported by the Grant-in-Aid for Scientific Research (C), grant number 26462515 (to K Oda) and Grant-in-Aid for Research Activity Start-up, grant number 25893229 (to Y Ikeda) from the Ministry of Education, Culture, Sports, Science, and Technology of Japan.

\section{Author details}

${ }^{1}$ Department of Obstetrics and Gynecology, The University of Tokyo, 7-3-1 Hongo, Bunkyo-ku, Tokyo 113-8655, Japan. ²Department of Obstetrics and Gynecology, Kawakita General Hospital, 1-7-3 Asagayakita, Suginami, Tokyo 166-0001, Japan. ${ }^{3}$ Department of Pathology, Kawakita General Hospital, 1-7-3 Asagayakita, Suginami, Tokyo 166-0001, Japan. ${ }^{4}$ Department of Pathology, Akita University Hospital, 44-2 Azahasunuma Hiroomote, Akita City 010-8502, Japan. ${ }^{5}$ Department of Pathology, The University of Tokyo, 7-3-1 Hongo, Bunkyo-ku, Tokyo 113-8655, Japan.

Received: 11 May 2014 Accepted: 31 August 2014

Published: 3 September 2014

\section{References}

1. Baird DD, Dunson DB, Hill MC, Cousins D, Schectman JM: High cumulative incidence of uterine leiomyoma in black and white women: ultrasound evidence. Am J Obstet Gynecol 2003, 188:100-107.

2. Stewart EA: Uterine fibroids. Lancet 2001, 357:293-298.

3. Seracchioli R, Rossi S, Govoni F, Rossi E, Venturoli S, Bulletti C, Flamigni C: Fertility and obstetric outcome after laparoscopic myomectomy of large myomata: a randomized comparison with abdominal myomectomy. Hum Reprod 2000, 15:2663-2668.

4. Cho FN: latrogenic abscess at uterine incision site after cesarean section: sonographic monitoring. J Clin Ultrasound 2008, 36:381-383.

5. Ghuman M, Rohlandt D, Joshy G, Lawrenson R: Post-caesarean section surgical site infection: rate and risk factors. N Z Med J 2011, 124:32-36.

6. LaMorte Al, Lalwani S, Diamond MP: Morbidity associated with abdominal myomectomy. Obstet Gynecol 1993, 82:897-900.

7. West S, Ruiz R, Parker WH: Abdominal myomectomy in women with very large uterine size. Fertil Steril 2006, 85:36-39. 
8. Rivlin ME, Patel RB, Carroll CS, Morrison JC: Diagnostic imaging in uterine incisional necrosis/dehiscence complicating cesarean section. J Reprod Med 2005, 50:928-932.

9. Obata-Yasuoka M, Ba-Thein W, Tsukamoto T, Yoshikawa H, Hayashi H: Vaginal Escherichia coli share common virulence factor profiles, serotypes and phylogeny with other extraintestinal E. coli. Microbiology 2002 $148: 2745-2752$.

doi:10.1186/1472-6874-14-104

Cite this article as: Oda et al.: Huge pyogenic cervical cyst with endometriosis, developing 13 years after myomectomy at the lower uterine segment: a case report. BMC Women's Health 2014 14:104.

\section{Submit your next manuscript to BioMed Central and take full advantage of:}

- Convenient online submission

- Thorough peer review

- No space constraints or color figure charges

- Immediate publication on acceptance

- Inclusion in PubMed, CAS, Scopus and Google Scholar

- Research which is freely available for redistribution 